\title{
The association between Helicobacter pylori seropositivity and risk of new-onset diabetes: a prospective cohort study
}

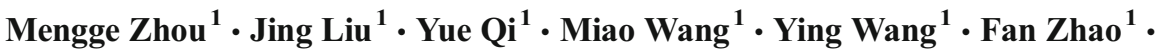 \\ Yongchen $\mathrm{Hao}^{1}$ • Dong Zhao ${ }^{1}$
}

Received: 10 June 2017 / Accepted: 31 August 2017 /Published online: 30 October 2017

(C) The Author(s) 2017. This article is an open access publication

\begin{abstract}
Aims/hypothesis Previous studies have suggested a possible connection between Helicobacter pylori (H. pylori) infection and diabetes risk. However, prospective studies examining direct associations between these two factors are relatively lacking. In this prospective cohort study, we aimed to evaluate the association between $H$. pylori infection and risk of developing diabetes.

Methods We performed a population-based prospective study, recruiting participants aged 45-74 years and without diabetes from the Chinese Multi-provincial Cohort Study in 2002, with a 10 year follow-up to investigate development of diabetes. $H$. pylori serostatus was determined by measuring serum H. pylori antibodies. H. pylori seropositivity was defined as the antibody concentration $\geq 10 \mathrm{U} / \mathrm{ml}$. To examine the association between $H$. pylori seropositivity and diabetes risk, modified Poisson regression was performed.

Results Of 2085 participants without diabetes, 1208 (57.9\%) were $H$. pylori seropositive in 2002. After multivariate adjustment of possible diabetes risk factors, $\mathrm{H}$. pylori seropositivity was associated with lower risk of diabetes (RR 0.78 [95\% CI $0.63,0.97], p=0.022$ ). Of the 1275 participants with $H$. pylori antibody measurements in both 2002 and 2007, 677 (53.1\%)
\end{abstract}

Electronic supplementary material The online version of this article (https://doi.org/10.1007/s00125-017-4465-2) contains peer-reviewed but unedited supplementary material, which is available to authorised users.

Dong Zhao

deezhao@vip.sina.com

1 Department of Epidemiology, Beijing Anzhen Hospital, Capital Medical University, Beijing Institute of Heart, Lung and Blood Vessel Diseases, the Key Laboratory of Remodelling-Related Cardiovascular Diseases, Ministry of Education, No. 2 Anzhen Road, Chaoyang District, Beijing 100029, China were persistently seropositive. A lower risk of diabetes was also observed in participants with persistent $H$. pylori seropositivity (RR 0.61 [95\% CI 0.41, 0.93], $p=0.020$ ), compared with those persistently seronegative.

Conclusions/interpretation H. pylori seropositivity was associated with lower risk of diabetes in this prospective cohort study. Extrapolation of these results and the mechanism underlying the observed association require further investigation.

Keywords Diabetes $\cdot$ Helicobacter pylori $\cdot$ Infection · Prospective cohort study $\cdot$ Seropositivity

$\begin{array}{ll}\text { Abbreviations } \\ \text { CagA } & \text { Cytotoxin-associated gene A } \\ \text { FBG } & \text { Fasting blood glucose } \\ \text { HDL-C } & \text { HDL-cholesterol } \\ \text { Hs-CRP } & \text { High-sensitivity C-reactive protein } \\ \text { IFG } & \text { Impaired fasting glucose } \\ \text { IQR } & \text { Interquartile range } \\ \text { LDL-C } & \text { LDL-cholesterol } \\ \text { PAI } & \text { Pathogenicity island }\end{array}$

\section{Introduction}

It is estimated that more than $50 \%$ of the world's population are infected with Helicobacter pylori, a gram-negative bacterium primarily colonising the human stomach [1]. The harmful effects of $H$. pylori infection on several gastrointestinal diseases have been well established [2]. An increasing number of studies have also revealed that $H$. pylori infection may have a wider ranging impact on health through its extragastrointestinal effects [3]. Specifically, this includes an association between $H$. pylori infection and diabetes [4-6], a 
harmful metabolic disease with rapidly increasing prevalence worldwide, particularly over the past 30 years [7].

A large number of published studies, reviews and book chapters have reported associations between $H$. pylori infection and serum or gastric concentrations of various hormones $[2,8-10]$, such as insulin, glucagon-like peptide 1, leptin, ghrelin, gastrin and somatostatin, some of which are involved in glucose metabolism directly or indirectly [11-17]. These studies provided the preliminary pathophysiological evidence for the potential connection between H. pylori infection and diabetes risk. Several studies have reported direct evidence for the association between $H$. pylori infection and diabetes risk [4-6]. However, most of these studies were based on relatively small sample sizes using either a cross-sectional or casecontrol design, with inconsistent results.

Whether H. pylori infection is indeed associated with diabetes risk is critically important and relevant to clinical decisions regarding future treatment of $H$. pylori as well as diabetes prevention strategies. Therefore, the purpose of this study was to examine the association between $H$. pylori infection and the long-term risk of developing diabetes based on a prospective cohort study with a relatively large sample size and multiple H. pylori test results.

\section{Methods}

Study population Study participants were recruited from the Chinese Multi-provincial Cohort Study; a community-based cohort study from 1992 [18]. Initially, 2349 of 2505 participants without diabetes, from two communities in Beijing, China, with blood samples obtained in 2002 for $H$. pylori measurement were enrolled in this study. We excluded 45 (1.9\%) deaths not related to diabetes and 219 (9.3\%) participants who were lost to follow-up. Ultimately, data from 2085 (88.8\%) individuals that had participated in either the 2007 $(n=1945)$ or $2012(n=1654)$ survey for diabetes were eligible for analysis to explore the association between $H$. pylori and the 10 year risk of developing diabetes (2002-2012; ESM Fig. 1a). Among these 2085 participants, 1728 had blood samples collected in 2007 for a second measurement of H. pylori antibodies. To evaluate the association between persistent $H$. pylori seropositivity (defined as participants testing seropositive to $H$. pylori in both 2002 and 2007) and risk of diabetes in 2012, 1275 participants without diabetes in both 2002 and 2007 with two H. pylori testing results were selected (ESM Fig. 1b).

The baseline (2002) characteristics of the recruited participants and participants lost to follow-up were compared (ESM Table 1). There was no statistically significant difference in the $H$. pylori seropositivity rate between the two groups.
The study was approved by the Ethics Committee of Beijing Anzhen Hospital. Participants provided written informed consent during the 2002, 2007 and 2012 surveys.

Establishment of $\boldsymbol{H}$. pylori serostatus $H$. pylori antibody concentrations were evaluated using previously frozen $\left(-80^{\circ} \mathrm{C}\right)$ serum samples obtained in 2002 and 2007 without freeze-thaw cycles. We measured serum $H$. pylori antibodies in samples collected in 2002 from all 2085 participants. Among the 2085 participants, 1275 participants had samples available for further measurement of $H$. pylori antibodies in 2007. All H. pylori antibodies were measured by latexenhanced turbidimetric immunoassay (Denka Seiken, Tokyo, Japan). H. pylori seropositivity was defined as H. pylori antibodies $\geq 10 \mathrm{U} / \mathrm{ml}$ with a sensitivity of $94.0 \%$ and specificity of $91.7 \%$ when using the endoscopic gastric mucosal atrophy and rapid urease test as the gold standard [19]. Persistent seropositivity was defined as $H$. pylori antibodies $\geq 10 \mathrm{U} / \mathrm{ml}$ in both the 2002 and 2007 samples for the same person.

Pre-study validation of the $H$. pylori antibody assay was performed by measuring $H$. pylori antibody in four quality control serum samples of known low-concentrations and four known high-concentrations each day for 5 consecutive days (ESM Fig. 2). Variation in detection of $H$. pylori antibodies was small in the pre-study. Alongside measurements of H. pylori antibody in the study population, six quality control serum samples were added at different positions in each batch, including three known low- and three known highconcentration $H$. pylori antibody samples. The mean coefficient of variation was $9.98 \%$ for the low-concentration H. pylori antibody controls and $3.26 \%$ for the highconcentration $H$. pylori antibody controls. All measurements were completed using the automatic biochemical analyser (Hitachi 7180, Hitachi, Tokyo, Japan).

Establishment of diabetes status Information regarding diabetes status was obtained from interview during on-site surveys, fasting serum glucose measurements on the 2007 or 2012 survey day and death reports during the follow-up period. The surveys in 2007 and 2012 were conducted face-to-face by specially trained investigators. Whether individuals had received a formal diagnosis of diabetes by a physician and whether the individuals had used glucose-lowering medications during the past 2 weeks was recorded in a standardised questionnaire. Fasting glucose concentrations were measured on fresh overnight fasting blood samples on the day of serum collection during both the 2002 and 2007 surveys by enzymatic methods (2002: Daiichi Pure Chemicals, Tokyo, Japan; 2007: HUMAN, Wiesbaden, Germany). Deaths were identified at follow-up visits by staff from collaborating centres and by regular searching of the death registration database of Beijing, which includes all deaths in the Beijing population. Diabetes was 
defined as any of the following criteria: (1) self-report of a physician's diagnosis of diabetes during the face-to-face survey, (2) use of glucose-lowering medications during the past 2 weeks, (3) fasting blood glucose (FBG) $\geq 7.0 \mathrm{mmol} / \mathrm{l}$ or (4) diabetes listed as the underlying cause of death on a death report.

Assessment of relevant covariates Other information, including demographic variables, level of education, smoking status and personal medical history, was collected in 2002 and 2007 [20]. Level of education was divided into two categories according to whether the participants received a college education (education time $\leq 12$ or $>12$ years). Smoking was defined as smoking one or more cigarettes per day for at least 3 months. Lipid-lowering therapies were recorded based on whether the participants were taking lipid-lowering medication.

Physical examinations, including height, weight and BP were performed by trained physicians during the on-site surveys in 2002 and 2007. BMI was calculated as weight in kilograms divided by height squared in meters. BP was measured with a regular mercury sphygmomanometer on the right arm while seated after resting for at least $5 \mathrm{~min}$. The mean value of two (2002) or three (2007) consecutive readings was used. Hypertension was defined as systolic $\mathrm{BP} \geq 140 \mathrm{mmHg}$, diastolic $\mathrm{BP} \geq 90 \mathrm{mmHg}$ and/or current antihypertensive treatment.

Laboratory measurements, including total cholesterol, triacylglycerol, LDL-cholesterol (LDL-C) and HDL-cholesterol (HDL-C) levels, were measured using fresh samples on the same day as serum collection in 2002 or 2007. Total cholesterol and triacylglycerol levels were determined using enzymatic methods (2002: Daiichi Pure Chemicals; 2007: HUMAN). LDL-C and HDL-C levels were measured using a homogeneous assay (2002: Daiichi Pure Chemicals; 2007: HUMAN). High-sensitivity C-reactive protein (Hs-CRP) was measured in 2015 using serum obtained in 2002 and 2007 by a latex-enhanced turbidimetric immunoassay assay (Denka Seiken) on an automatic biochemical analyser (Hitachi 7180).

Sample power estimation One cohort study has investigated the association between $H$. pylori seropositivity and risk of diabetes [6]. This study reported that those who were seropositive for $H$. pylori at recruitment were 2.69 times to develop diabetes than seronegative individuals. In our study, the cumulative incidence of diabetes in 10 years was $13.6 \%$ in seronegative participants. Based on this information with an alpha (probability of type I error) of 0.05 , the actual sample size of 2085 in this study provided sufficient statistical power (power $=0.86$ ).

Statistical analysis Baseline characteristics of the participants are described as mean (SD) for continuous variables with a normal distribution, median (interquartile range, IQR) for continuous variables with a skewed distribution and percentages for categorical variables. Baseline characteristics of the participants with and without $H$. pylori seropositivity were compared using the $t$ test, Wilcoxon rank test or $\chi^{2}$ test.

To examine the association between $H$. pylori seropositivity and diabetes risk (2002-2012) or persistent H. pylori seropositivity and diabetes risk (2007-2012), modified Poisson regression was performed to estimate RR and robust standard errors to estimate the 95\% CIs [21]. Univariate analysis was performed first. Then, we performed a sex- and age-adjusted model (Model 1). We added further possible risk factors of diabetes to evaluate the association (Model 2), including BMI (continuous variable), HDL-C (continuous variable), triacylglycerol $(<1.7$ or $\geq 1.7 \mathrm{mmol} / \mathrm{l}$ ), FBG (continuous variable), systolic blood pressure (per $5 \mathrm{mmHg}$, continuous variable) and lipid-lowering medication (no or yes). Hs-CRP $(<3$ or $\geq 3 \mathrm{mg} / \mathrm{l}$ ), a sensitive biomarker of inflammation, reported to be associated with high risk of diabetes [22], was added in Model 3. Moreover, level of education was also included in the model (Model 4), since socioeconomic status was an important factor for $H$. pylori infection, and level of education was associated with socioeconomic status.

Since participants did not undergo OGTT at baseline, the study may have included participants with diabetes only detectable by OGTT, particularly among participants with impaired fasting glucose (IFG) [23]. Thus, we further excluded participants with IFG (FBG $>6.1 \mathrm{mmol} / \mathrm{l})$ from the sensitivity analysis.

Subgroup analyses were performed using baseline (2002) characteristics, including age ( $<60$ or $\geq 60$ years), sex (men or women), BMI $\left(<25\right.$ or $\left.\geq 25 \mathrm{~kg} / \mathrm{m}^{2}\right)$, HDL-C $(<1.0$ or $\geq 1.0 \mathrm{mmol} / \mathrm{l})$, triacylglycerol $(<1.7$ or $1.7 \mathrm{mmol} / \mathrm{l})$, Hs-CRP $(<3$ or $\geq 3 \mathrm{mg} / \mathrm{l}$ ), hypertension (no or yes), FBG ( $<5.6$ or $\geq 5.6 \mathrm{mmol} / \mathrm{l}$ ) and lipid-lowering therapy (no or yes) in a multivariable adjusted Poisson regression model. Alongside the grouping factors, other factors were used as adjustment variables. In addition, we performed further subgroup analyses using different baseline information (2002 and 2007) for the 5 year association between $H$. pylori and risk of diabetes. RRs between subgroups were compared using Z-test [24].

Statistical analyses were conducted by SAS 9.2 (SAS Institute, Cary, NC, USA). Two-tailed $p$ values $<0.05$ were considered statistically significant.

\section{Results}

Baseline characteristics of the study participants The mean age of the participants $(n=2085)$ was $57.0( \pm 7.8)$ years in 2002 and $1214(58.2 \%)$ participants were women. The $H$. pylori seropositivity rate was $57.9 \%$ in 2002 . Baseline (2002) characteristics of the study participants were compared according to H. pylori serostatus (Table 1). H. pylori seropositive participants were younger than seronegative participants and had a 
Table 1 Baseline (2002) characteristics of the study participants

\begin{tabular}{lllr}
\hline & $\begin{array}{l}\text { H. pylori seronegativity } \\
(n=877)\end{array}$ & $\begin{array}{l}\text { H. pylori seropositivity } \\
(n=1208)\end{array}$ & $p$ value \\
\hline Age, mean (SD), years & $58.0(7.8)$ & $56.3(7.8)$ & $<0.001$ \\
Women, $n$ (\%) & $509(58.0)$ & $705(58.4)$ & 0.883 \\
BMI, mean (SD), kg/m ${ }^{2}$ & $25.0(3.2)$ & $25.4(3.3)$ & 0.005 \\
Blood lipid levels, mean (SD), mmol/1 & & & \\
Total cholesterol & $5.4(1.0)$ & $5.3(1.0)$ & 0.060 \\
LDL-C & $3.3(0.8)$ & $3.2(0.8)$ & 0.373 \\
HDL-C & $1.41(0.33)$ & $1.36(0.30)$ & 0.001 \\
Triacylglycerol, median (IQR) & $1.3(0.9-1.9)$ & $1.3(0.9-1.9)$ & 0.775 \\
FBG, mean (SD), mmol/1 & $4.7(0.5)$ & $4.7(0.5)$ & 0.578 \\
BP, mean (SD), mmHg & & $129.4(18.4)$ & 0.204 \\
Systolic & $130.5(19.2)$ & $81.8(10.2)$ & 0.765 \\
Diastolic & $81.7(10.1)$ & $1.1(0.6-2.2)$ & 0.225 \\
Hs-CRP, median (IQR), mg/1 & $1.1(0.6-2.2)$ & $143(11.8)$ & 0.472 \\
Current smoking, $n(\%)$ & $113(12.9)$ & $495(41.0)$ & 0.003 \\
Hypertension, $n(\%)$ & $416(47.4)$ & $82(6.8)$ & 0.456 \\
Lipid-lowering medication, $n(\%)$ & $67(7.6)$ & $526(42.5)$ & 0.038 \\
Education $\leq 12$ years, $n(\%)$ & $342(39.0)$ & & \\
\hline
\end{tabular}

${ }^{\text {a }}$ There is a statistical difference in HDL-C between $H$. pylori seronegative and H. pylori seropositive groups. We have retained two decimal places for this data to make this difference clear significantly higher BMI and lower serum HDL-C and a lower proportion had hypertension. The proportion of participants receiving college education ( $\geq 12$ years) was higher among the $H$. pylori seronegative group. There were no significant differences in other characteristics between the H. pylori seropositive and seronegative groups.

Association between $\boldsymbol{H}$. pylori seropositivity and risk of
developing diabetes During the follow-up period (20022012), 259 (12.4\%) new cases of diabetes were recorded. Among these participants, $140(11.6 \%)$ were identified in the $H$. pylori seropositive group and $119(13.6 \%)$ were in the seronegative group. Table 2 displays the RRs and 95\% CIs for the association between $H$. pylori seropositivity and diabetes risk. Using univariate analysis, $H$. pylori was associated with a reduced risk of diabetes (RR 0.85 [95\% CI 0.68, 1.07]) but without statistical significance $(p=0.176)$. The $\mathrm{RR}$ value was almost unchanged after sex- and age-adjustment (RR 0.86 [95\% CI 0.68, 1.08], $p=0.200$ ). After multivariate adjustment for possible diabetes risk factors, H. pylori seropositivity was associated with a significantly lower risk of diabetes (RR 0.78 [95\% CI 0.63, 0.97], $p=0.025$ ). This RR value was unchanged after further adjustment for Hs-CRP (RR 0.78 [95\% CI 0.63, 0.97], $p=0.025$ ) and level of education (RR 0.78 [95\% CI 0.63, 0.97]; $p=0.022$ ).

After further excluding participants with IFG, the association between $H$. pylori and diabetes risk was similar (Table 2; details in ESM Table 2).
Association between persistent $\boldsymbol{H}$. pylori seropositivity and risk of developing diabetes A total of 1275 participants had H. pylori antibody measurements in both 2002 and 2007, of which $677(53.1 \%)$ were persistently seropositive and $514(40.3 \%)$ were persistently seronegative. Only 19 (1.5\%) participants transformed from seronegativity to seropositivity and $65(5.1 \%)$ from seropositivity to seronegativity. A total of 84 individuals developed diabetes among these participants between 2007 and 2012, including 44 (8.6\%) among those who were persistently seronegative, 35 (5.2\%) among those who were persistently seropositive and five (7.7\%) among those who transformed from seropositive to seronegative. No new cases of diabetes were identified among those transformed from seronegative to seropositive. As the serostatus only changed in a very small number of participants, we compared the risk of developing diabetes in participants who were persistently seropositive with those who were persistently seronegative, with 2007 as the baseline. Therefore, 1191 participants were included in the analysis of the association between persistent H. pylori seropositivity and diabetes risk. The baseline (2007) characteristics of these participants were compared across H. pylori serostatus (ESM Table 3). As displayed in Table 3 (details in ESM Table 4), persistent H. pylori seropositivity was also associated with a lower risk of developing diabetes in both univariate and multivariate analyses. After excluding participants with IFG, H. pylori seropositivity was still associated with lower risk of diabetes (Table 3 ). 
Table 2 Association between H. pylori infection and risk of developing diabetes (2002-2012)

\begin{tabular}{llllll}
\hline & \multicolumn{2}{l}{ All participants $(n=2085)$} & & \multicolumn{2}{l}{ Participants without IFG $(n=2043)$} \\
\cline { 2 - 3 } \cline { 5 - 6 } & RR $(95 \%$ CI $)$ & $p$ value & & RR $(95 \%$ CI $)$ & $p$ value \\
\hline Events No. & 259 & & & 235 & \\
Unadjusted & $0.85(0.68,1.07)$ & 0.176 & & $0.86(0.67,1.09)$ & 0.215 \\
Model 1 & $0.86(0.68,1.08)$ & 0.200 & & $0.86(0.67,1.10)$ & 0.223 \\
Model 2 & $0.78(0.63,0.97)$ & 0.025 & & $0.80(0.64,1.01)$ & 0.059 \\
Model 3 & $0.78(0.63,0.97)$ & 0.025 & & $0.79(0.63,0.99)$ & 0.043 \\
Model 4 & $0.78(0.63,0.97)$ & 0.022 & & $0.80(0.64,1.01)$ & 0.057 \\
\hline
\end{tabular}

Model 1: Sex- and age-adjusted

Model 2: Model 1 + BMI (continuous variable), systolic BP (per $5 \mathrm{mmHg}$, continuous variable), HDL-C (continuous variable), triacylglycerol $(<1.7$ or $\geq 1.7 \mathrm{mmol} / \mathrm{l}$ ), FBG (continuous variable), lipid-lowering therapy (no or yes)

Model 3: Model $2+$ Hs-CRP $(<3$ or $\geq 3 \mathrm{mg} / \mathrm{l})$

Model 4: Model $3+$ level of education ( $\leq 12$ or $>12$ years)

No., number

\section{Subgroup analysis for the association between $H$. pylori} seropositivity and risk of developing diabetes The associations between $H$. pylori seropositivity and risk of developing diabetes were evaluated based on different subgroups, including age ( $<60$ or $\geq 60$ years), sex (men or women), BMI $(<25$ or $\left.\geq 25 \mathrm{~kg} / \mathrm{m}^{2}\right)$, HDL-C $(<1.0$ or $\geq 1.0 \mathrm{mmol} / \mathrm{l})$, triacylglycerol $(<1.7$ or $\geq 1.7 \mathrm{mmol} / \mathrm{l})$, Hs-CRP $(<3$ or $\geq 3 \mathrm{mg} / \mathrm{l})$, hypertension (no or yes), FBG $(<5.6$ or $\geq 5.6 \mathrm{mmol} / \mathrm{l}$ ) and lipid-lowering therapy (no or yes) (Fig. 1). H. pylori seropositivity was associated with lower risk of developing diabetes in different subgroups except in participants receiving lipid-lowering medication (RR 1.11 [95\% CI 0.64, 1.94]). In addition, H. pylori seropositivity was associated with a lower 5 year risk of developing diabetes based on different baseline information (2002-2007; 2007-2012).

\section{Discussion}

In this prospective cohort study, we carefully examined the association between $H$. pylori seropositivity and risk of developing diabetes, based on reliable measurements of antibody against H. pylori and a clear diagnosis of diabetes. We determined that H. pylori seropositivity was associated with lower risk of developing diabetes. To our knowledge, this is the first cohort study to evaluate the association between persistent $H$. pylori seropositivity and risk of developing diabetes and the first study to detect an inverse association between these factors.

The literature regarding the association between $\mathrm{H}$. pylori and diabetes risk is inconsistent, with reports of positive, null and negative associations [4-6]. A published meta-analysis systematically reviewed the association between $H$. pylori
Table 3 Association between persistent $H$. pylori infection and risk of developing diabetes (2007-2012)

\begin{tabular}{|c|c|c|c|c|}
\hline & \multicolumn{2}{|c|}{ All participants $(n=1191)$} & \multicolumn{2}{|c|}{ Participants without IFG ( $n=1036)$} \\
\hline & RR $(95 \% \mathrm{CI})$ & $p$ value & $\mathrm{RR}(95 \% \mathrm{CI})$ & $p$ value \\
\hline Events No. & 79 & & 37 & \\
\hline Unadjusted & $0.60(0.39,0.93)$ & 0.021 & $0.52(0.27,0.98)$ & 0.044 \\
\hline Model 1 & $0.58(0.37,0.89)$ & 0.013 & $0.50(0.26,0.98)$ & 0.044 \\
\hline Model 2 & $0.62(0.41,0.92)$ & 0.019 & $0.50(0.26,0.99)$ & 0.047 \\
\hline Model 3 & $0.61(0.41,0.91)$ & 0.017 & $0.51(0.25,1.01)$ & 0.054 \\
\hline Model 4 & $0.61(0.41,0.93)$ & 0.020 & $0.50(0.26,0.99)$ & 0.048 \\
\hline
\end{tabular}

Persistent seropositivity: individuals were seropositive in both 2002 and 2007

Model 1: Sex- and age-adjusted

Model 2: Model 1 + BMI (continuous variable), systolic BP (per $5 \mathrm{mmHg}$, continuous variable), HDL-C (continuous variable), triacylglycerol $(<1.7$ or $\geq 1.7 \mathrm{mmol} / \mathrm{l})$, FBG (continuous variable), lipid-lowering therapy (no or yes)

Model 3: Model $2+$ Hs-CRP $(<3$ or $\geq 3 \mathrm{mg} / \mathrm{l})$

Model 4: Model $3+$ level of education ( $\leq 12$ or $>12$ years)

No., number 
Fig. 1 Subgroup analyses for the association between $H$. pylori seropositivity and risk of developing diabetes. All RRs were adjusted for age (continuous variable), sex (men or women), BMI (continuous variable), systolic BP (per $5 \mathrm{mmHg}$, continuous variable), HDL-C (continuous variable), triacylglycerol $(<1.7$ or $\geq 1.7 \mathrm{mmol} / \mathrm{l}$ ), FBG (continuous variable), lipid-lowering therapy (no or yes) and Hs-CRP ( $<3$ or $\geq 3 \mathrm{mg} / \mathrm{l})$, except for the grouping variable

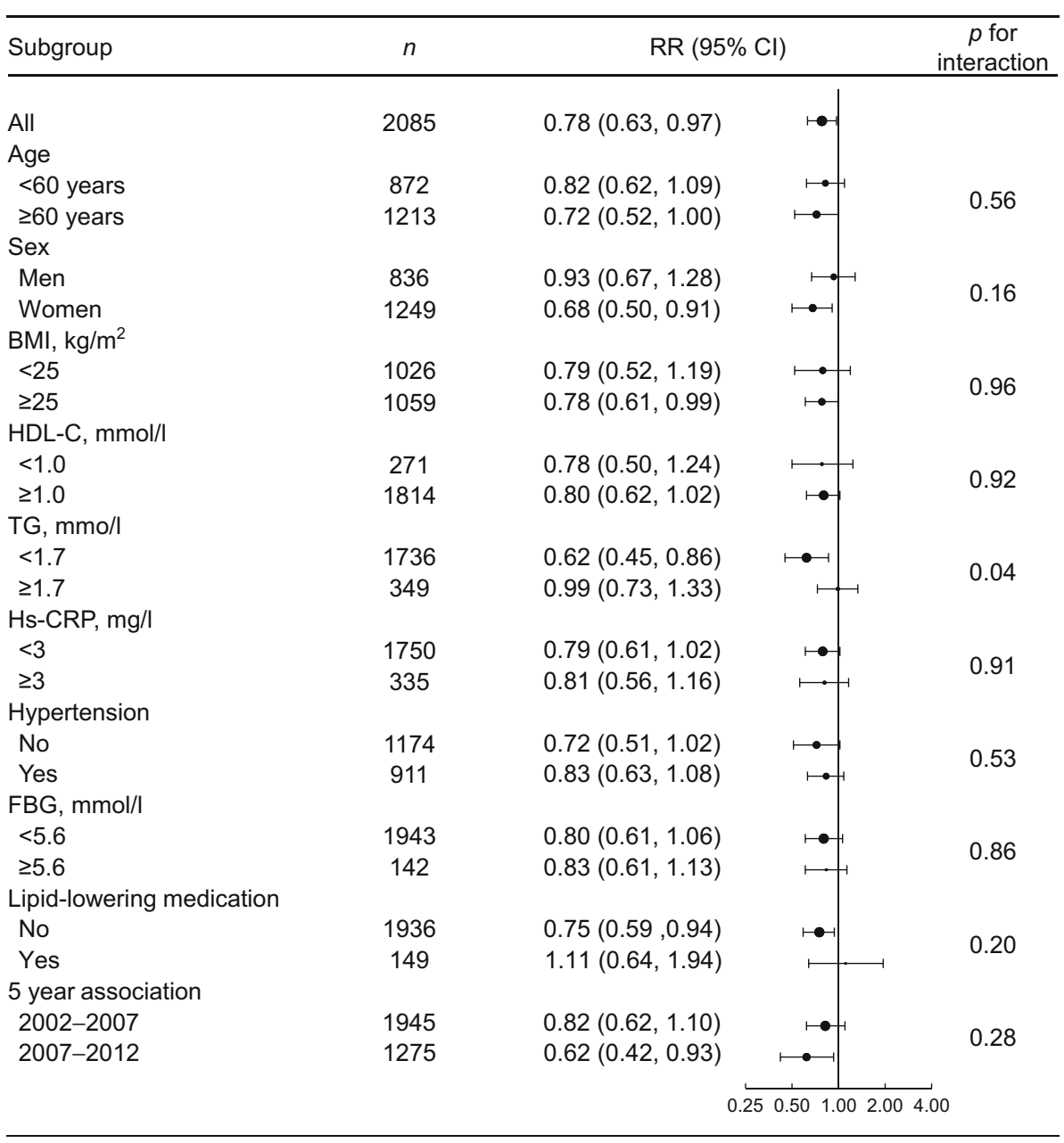

infection and diabetes based on observational studies and drew the conclusion that there was a trend toward more frequent $H$. pylori infection in individuals with diabetes [4]. However, cross-sectional or case-control studies could not determine the causality between $H$. pylori infection and diabetes. We are aware of only one prospective cohort study evaluating the association between $H$. pylori seropositivity and risk of diabetes [6]. This study derived data from the Sacramento Area Latino Study on Ageing and found that H. pylori seropositivity was associated with increased risk of incident diabetes (HR 2.7 [95\% CI 1.1, 6.6]). However, the characteristics of our study population differed from this study in that their participants were older (60 to 101 years old) and the $H$. pylori seropositivity rate was much higher than ours (91.9\% vs $57.9 \%)$.

Our finding of an inverse association between $H$. pylori seropositivity and diabetes risk was of physiological relevance. Several studies have reported that $H$. pylori seropositivity was associated with different hormones [2, 8-10], most of which were involved in glucose metabolism directly or indirectly [11-17]. To our knowledge, at least three hormones supported our results, including gastrin, leptin and ghrelin.
Studies have reported that long-term gastrin treatment resulted in improved metabolic control and exerted proliferative effects on pancreatic beta cells [11]. Leptin has been reported to reduce hyperglycaemia in rodent models of type 1 diabetes and has recently been shown to normalise fasting plasma glucose concentrations in a rodent model of polygenic obesity and type 2 diabetes [14]. In contrast, ghrelin has been shown to upregulate systemic glucose levels in both humans and rodents [16]. Thus, studies reporting elevated gastrin and leptin and decreased ghrelin levels in individuals infected with H. pylori support our findings. However, the regulation of these hormones is intricate. Therefore, the association between $H$. pylori infection, hormones and diabetes requires further studies.

H. pylori has co-existed with humans for at least 58,000 years [25], and only $10 \%$ of asymptomatic infected individuals develop gastrointestinal disease during their lifetime [1]. Regarding the view of co-adaptation of humans and microorganisms, we might reconsider our definitions of H. pylori and perhaps recognise it as a normal member of the human gastric microbiome [26]. The Kyoto global consensus on $H$. pylori gastritis, published in 2015, strongly 
recommended that all individuals infected with $H$. pylori should be offered eradication therapy, unless there were competing considerations [27]. If $H$. pylori infection could indeed affect diabetes risk, we should reconsider this strategy. More studies are needed to explore the association between these factors.

Several limitations associated with the current study deserve mention. First, 45 participants died and 219 were lost at followup between 2002 and 2012, which raised a consideration for the competing risk. To test each extreme situation, we analysed the association between $H$. pylori infection and risk of developing diabetes assuming that all of the participants who died or were lost at follow-up did not develop diabetes and then again, but assuming that all did develop diabetes (ESM Table 5). In both of these analyses, $H$. pylori seropositivity was still associated with lower risk of diabetes. Therefore, we believe that loss and death in our study have little effect on our findings. Additionally, time-to-event analysis could not be performed in our study as we only collected information regarding the development of diabetes at 5 yearly intervals. Furthermore, we did not collect the information about $H$. pylori eradication treatments in our study. However, as we knew, the awareness rate of $H$. pylori was very low even among individuals with gastrointestinal symptoms in China [28], so we could speculate that the treatment rate could also be very low. In addition, we detected H. pylori in both 2002 and 2007 and found that the serostatus changed in only a few participants. To some extent, multi-point detection might better explain the long-term relationship between $H$. pylori and humans. In addition, we did not specifically measure the concentrations of antibodies against cytotoxin-associated gene $\mathrm{A}(\mathrm{CagA})$, a major factor influencing the virulence of $H$. pylori, in our population. A study has reported that FBG levels were significantly higher in uninfected mice compared with $H$. pylori-infected mice, regardless of the strain of H. pylori utilised [29]. However, only mice infected with a Cag pathogenicity island (PAI)-negative H. pylori strain, but not with an isogenic Cag PAI-positive strain, showed improvements in glucose tolerance. Therefore, further studies are still needed to investigate the association between antibodies against CagA and glycometabolism.

In summary, these findings provide new direct evidence for the association between $H$. pylori infection and diabetes risk. Analysis of the association between H. pylori and diabetes is of great public health and clinical significance given the high prevalence of H. pylori infection and significant burden of diabetes. Thus, clarification of the association between these factors might influence clinical decisions regarding future treatment of $H$. pylori infection as well as diabetes prevention strategies. However, extrapolation of this study requires verification by other prospective studies and clinical trials, and the underlying mechanism warrants further investigation.
Acknowledgements Denka Seiken Co., Ltd. (Tokyo, Japan) provided all $H$. pylori antibody detection kits in this study, but they were not involved in the study design, data analysis or result interpretation.

Data availability The datasets generated during and/or analysed during the current study are not publicly available due to ethical constraints in consideration of participants' privacy and intellectual property protection but are available from the corresponding author on reasonable request.

Funding Participants recruitment and data collection in this study were supported by Beijing Municipal Science \& Technology Commission (953850700), the National Science \& Technology Pillar Program during the Eleventh Five-Year Plan Period (2006BAI01A01 and 2006BAI01A02) and the National Science \& Technology Pillar Program during the Twelfth Five-Year Plan Period (2011BAI09B01). The study design, data analysis, interpretation and other pertinent aspects were funded by the National Natural Science Foundation of China (81170266, 81070226 and 81570409), Beijing, China.

Duality of interest The authors declare that there is no duality of interest associated with this manuscript.

Contribution statement MGZ and DZ analysed the data and drafted the manuscript. All authors qualify for authorship according to International Committee of Medical Journal Editors criteria. They have all contributed to the conception and design of the study, the interpretation of the data, the critical revision of the article for important intellectual content and the final approval of the version to be published. DZ is responsible for the integrity of the work as a whole.

Open Access This article is distributed under the terms of the Creative Commons Attribution 4.0 International License (http:// creativecommons.org/licenses/by/4.0/), which permits unrestricted use, distribution, and reproduction in any medium, provided you give appropriate credit to the original author(s) and the source, provide a link to the Creative Commons license, and indicate if changes were made.

\section{References}

1. McColl KE (2010) Helicobacter pylori infection. N Engl J Med 362:1597-1604

2. Hidekazu S, Robin W, Barry M (2016) Helicobacter pylori. Springer Japan, Japan

3. Franceschi F, Zuccala G, Roccarina D, Gasbarrini A (2014) Clinical effects of Helicobacter pylori outside the stomach. Nat Rev Gastroenterol Hepatol 11:234-242

4. Zhou X, Zhang C, Wu J, Zhang G (2013) Association between Helicobacter pylori infection and diabetes mellitus: a metaanalysis of observational studies. Diabetes Res Clin Pract 99: 200-208

5. Haj S, Raviv M, Muhsen K (2016) Helicobacter pylori infection and diabetes mellitus. In: Bruna MR (eds) Extradigestive manifestations of Helicobacter pylori infection-an overview, Dr B Roesler (Ed.), InTech, https://doi.org/10.5772/63826

6. Jeon CY, Haan MN, Cheng C et al (2012) Helicobacter pylori infection is associated with an increased rate of diabetes. Diabetes Care 35:520-525

7. Zhou B, Lu Y, Hajifathalian K et al (2016) Worldwide trends in diabetes since 1980: a pooled analysis of 751 population-based studies with 4.4 million participants. Lancet 387:1513-1530 
8. Polyzos SA, Kountouras J, Zavos C, Deretzi G (2011) The association between Helicobacter pylori infection and insulin resistance: a systematic review. Helicobacter 16:79-88

9. Yap TW, Leow AH, Azmi AN et al (2015) Changes in metabolic hormones in Malaysian young adults following Helicobacter pylori eradication. PLoS One 10:e0135771

10. Kaneko H, Konagaya T, Kusugami K (2002) Helicobacter pylori and gut hormones. J Gastroenterol 37:77-86

11. Tellez N, Montanya E (2014) Gastrin induces ductal cell dedifferentiation and beta-cell neogenesis after $90 \%$ pancreatectomy. J Endocrinol 223:67-78

12. Sliwinska-Mosson M, Vesely M, Milnerowicz H (2014) The clinical significance of somatostatin in pancreatic diseases. Ann Endocrinol-Paris 75:232-240

13. Dezaki K (2013) Ghrelin function in insulin release and glucose metabolism. Endocr Dev 25:135-143

14. Cummings BP (2013) Leptin therapy in type 2 diabetes. Diabetes Obes Metab 15:607-612

15. Meier JJ (2012) GLP-1 receptor agonists for individualized treatment of type 2 diabetes mellitus. Nat Rev Endocrinol 8:728-742

16. Yada T, Damdindorj B, Rita RS et al (2014) Ghrelin signalling in beta-cells regulates insulin secretion and blood glucose. Diabetes Obes Metab 16(Suppl 1):111-117

17. Drucker DJ (2007) The role of gut hormones in glucose homeostasis. J Clin Invest 117:24-32

18. Liu J, Hong Y, D’Agostino RB Sr et al (2004) Predictive value for the Chinese population of the Framingham CHD risk assessment tool compared with the Chinese Multi-Provincial Cohort Study. JAMA 291:2591-2599

19. Furuta $\mathrm{T}$ (2016) Evaluation of the novel anti-H. pylori antibody detection kit for a clinical chemistry auto-analyzer. Jpn J Med Pharm Sci 73:451-457 [article in Japanese]
20. Liu J, Wang W, Qi Y et al (2014) Association between the lipoprotein-associated phospholipase A2 activity and the progression of subclinical atherosclerosis. J Atheroscler Thromb 21:532-542

21. Lumley T, Kronmal R, Ma S (2006) Relative risk regression in medical research: models, contrasts, estimators, and algorithms. UW Biostatistics Working Paper Series. Working paper 293. Available from http://biostats.bepress.com/uwbiostat/paper293

22. Wang X, Bao W, Liu J et al (2013) Inflammatory markers and risk of type 2 diabetes: a systematic review and meta-analysis. Diabetes Care 36:166-175

23. Liu J, Zhao D, Liu J, Qi Y, Sun J, Wang W (2013) Prevalence of diabetes mellitus in outpatients with essential hypertension in China: a cross-sectional study. BMJ Open 3:e003798

24. Altman DG, Bland JM (2003) Interaction revisited: the difference between two estimates. BMJ 326:219

25. Linz B, Balloux F, Moodley Y et al (2007) An African origin for the intimate association between humans and Helicobacter pylori. Nature 445:915-918

26. Atherton JC, Blaser MJ (2009) Coadaptation of Helicobacter pylori and humans: ancient history, modern implications. J Clin Invest 119:2475-2487

27. Sugano K, Tack J, Kuipers EJ et al (2015) Kyoto global consensus report on Helicobacter pylori gastritis. Gut 64:1353-1367

28. Dong J (2015) Gastroscopy for Hpylori infection awareness survey analysis. China Health Ind 2015 09:176-177

29. Bassaganya-Riera J, Dominguez-Bello MG, Kronsteiner B et al (2012) Helicobacter pylori colonization ameliorates glucose homeostasis in mice through a PPAR gamma-dependent mechanism. PLoS One 7:e50069 